\title{
Role of the signs of obturator hernia in clinical practice: Author's reply
}

\author{
Konstantinos Apostolou ${ }^{1}\left[\right.$. Dimitrios Schizas ${ }^{1}$
}

Received: 19 October 2020 / Accepted: 10 November 2020 / Published online: 23 November 2020

○) Springer-Verlag France SAS, part of Springer Nature 2020

The authors would like to thank Yale et al. for their precise and productive comments on the standardization in reporting obturator's hernia specific signs, namely Howship-Romberg and Hannington-Kiff sign, as stated in their letter to the editor entitled "Role of the signs of obturator hernia in clinical practice" [1]. Unfortunately, the method of performing and assessing the presence or absence of the Howship-Romberg sign was not reported in any of the studies included. It would be interesting to see if the positivity of that sign following a standardization in its performance is going to change in future studies reviewing this topic. Additionally, reports on the Hannington-Kiff sign in patients suffering from an obturator hernia are sparse in the literature, despite some studies identifying it as more sensitive and specific, compared to the Howship-Romberg sign [2].

However, the authors would like to highlight the crucial role of computed tomography scan in the accurate and timely diagnosis of an obturator hernia, with its diagnostic accuracy having been demonstrated to be up to $85 \%$ in the literature [3]. Abdominal computed tomography scan was the most common modality used for the diagnosis $(84.2 \%$ of cases), with its performance having been associated with a significantly decreased peri-operative mortality rate (Odds ratio $=0.29$ ). Considering the clinical symptoms and signs of obturator hernia patients, including bowel obstruction in nearly all patients (93.8\%) as well as abdominal pain and vomiting in the majority of them, computed tomography ranks first in the algorithm of an accurate preoperative diagnosis, which allows a more directed surgical operation instead of an exploratory laparotomy. According to authors' personal experience, although obturator's hernia specific symptoms and signs are extremely useful in the clinical setting, the lack of standardization in their performance or even

Konstantinos Apostolou

konstantinos.apostolou@gmail.com

1 First Department of Surgery, Laikon General Hospital, National and Kapodistrian University of Athens, Ag. Thoma 17 Str., Goudi, 11527 Athens, Greece their negativity should not be scrutinized, as the majority of these patients presents with acute onset abdominal pain and bowel obstruction and as a result will eventually undergo an abdominal computed tomography scan. Thus, clinical experience along with high clinical suspicion for the possible existence of an obturator hernia, especially when combined with a computed tomography scan, may outshine the diagnostic accuracy of a non-standardized performance of Howship-Romberg and Hannington-Kiff sign in contemporary medical practice.

\section{Compliance with ethical standards}

Conflict of interest The authors have no conflicts of interest to disclose.

\section{References}

1. Yale SH, Tekiner H, Yale ES (2020) Role of the signs of obturator hernia in clinical practice. Hernia. https://doi.org/10.1007/s1002 9-020-02296-2

2. Karasaki T, Nakagawa T, Tanaka N (2014) Obturator hernia: the relationship between anatomical classification and the HowshipRomberg sign. Hernia 18(3):413-416. https://doi.org/10.1007/ s10029-013-1068-9

3. Chan KV, Chan CK, Yau KW, Cheung MT (2014) Surgical morbidity and mortality in obturator hernia: a 10-year retrospective risk factor evaluation. Hernia 18(3):387-392. https://doi. org/10.1007/s10029-013-1169-5

Publisher's Note Springer Nature remains neutral with regard to jurisdictional claims in published maps and institutional affiliations. 